\title{
Unusual manifestations of type II cryoglobulinaemia associated with Waldenström's macroglobulinaemia
}

\author{
P Blanco, J-F Viallard, J Rivel, B Leng, J-L Pellegrin
}

\begin{abstract}
Cryoglobulinaemia in association with Waldenström's macroglobulinaemia is relatively common, ranging from $8 \%$ to $18 \%$ of cases; however, $<5 \%$ have symptoms or complications. We describe a patient with a history of cutaneous, peritoneal, and fallopian tube vasculitis related to type II cryoglobulinaemia associated with Waldenström's macroglobulinaemia. Cytotoxic treatment was initiated (cyclophosphamide, vincristine, and prednisone) and had a good initial response. However, after the third course of chemotherapy, the patient presented with septic shock and died. Even though cryoglobulinaemia is a model of systemic vasculitis, peritoneal and fallopian tube vasculitis associated with type II cryoglobulinemia has not been described previously.

(f Clin Pathol 2000;53:882-884)
\end{abstract}

Keywords: cryoglobulinaemia; Waldenström's macroglobulinaemia; peritoneum; fallopian tube

First described in 1944, ${ }^{1}$ Waldenström's macroglobulinaemia is thought to be a lymphoproliferative disease characterised by a clonal proliferation of B cells or lymphoplasmacytic cells in the bone marrow and/or lymph nodes, and monoclonal IgM in the plasma. Cryoglobulinaemia in association with Waldenström's macroglobulinaemia is relatively common, ranging from $8 \%$ to $18 \%$ of cases. ${ }^{2}$ However, $<5 \%$ of patients have symptoms or complications. Its main clinical manifestations are systemic vasculitis, arthralgias, myalgias, vascular purpura, glomerulonephritis, peripheral neuropathy, and cardiac involvement ${ }^{3}$; gastrointestinal symptoms induced by cryoglobulinemia are unusual and peritoneal involvement has never been described. Here, we report a patient presenting with cutaneous, peritoneal, and fallopian tube vasculitis related to mixed cryoglobulinaemia associated with Waldenström's macroglobulinaemia.

Laboratoire d'Anatomopathologie, Hôpital Pellegrin,

Place

Amélie-Raba-Léon, 33076 Bordeaux,

France

J Rivel

Correspondence to: Dr Viallard

JF.Viallard@

umr5540.u-bordeaux2.fr

Accepted for publication 12 May 2000 Case report

A 57 year old white woman was referred to hospital because of a two week history of intermittent fever, fatigue, $5 \mathrm{~kg}$ weight loss, several nodular purpuric cutaneous lesions, arthalgias of her ankles, wrists, metacarpophalangeal joints and shoulders, abdominal pain with diarrhoea and vomiting. On physical examination, she was pale and pyrexial $\left(39^{\circ} \mathrm{C}\right)$. There was a palpable nodular purpura of the legs and no arthritis. A splenomegaly was present with a diffuse abdominal tendernesss but no contracture. There was no peripheral lymphadenopathy and neurological examination was normal. Routine haematological tests revealed: haemoglobin, $99 \mathrm{~g} /$ litre; white blood cell count, $4.4 \times$ $10^{9} /$ litre; platelets, $289 \times 10^{9} /$ litre, erythrocyte sedimentation rate, $107 \mathrm{~mm}$ in the first hour; and $\mathrm{C}$ reactive protein, $107 \mathrm{mg} /$ litre (normal, $<10$ ). Results of blood chemical tests were unremarkable (serum electrolytes, urea, creatinine). Serum protein was $50 \mathrm{~g} / \mathrm{litre}$ and immunoelectrophoresis disclosed an $\operatorname{Ig} M \kappa$ paraprotein. Bence-Jones protein was detected in the urine and serum immunoglobulin values were as follows: IgG, $0.24 \mathrm{~g} /$ litre; IgM, $1.09 \mathrm{~g} /$ litre; IgA, $0.28 \mathrm{~g} /$ litre. Immunological studies (antinuclear antibodies, antineutrophil cytoplasm antibodies) were negative except for the presence of rheumatoid factors. C4 concentrations were dramatically low $(0.07 \mathrm{~g} /$ litre; normal range, $0.2-0.4 \mathrm{~g} /$ litre). Serological tests for virus infection were negative (Epstein-Barr virus, cytomegalovirus, hepatitis $\mathrm{B}$ and $\mathrm{C}$ virus, human immunodeficiency virus 1 and 2). There was a mixed cryoglobulinaemia $(35 \mathrm{mg} /$ litre) with an $\operatorname{IgM}$ monoclonal component. The IgM was an autoantibody with rheumatoid factor activity. Culture of blood and urine specimens yielded no microorganism. A bone marrow aspirate demonstrated infiltration with lymphocytoid plasma cells and mature plasma cells. Chest and abdominal computed tomography (CT) disclosed mediastinal, paratracheal, and retroperitoneal lymphadenopathies and a splenomegaly. In addition, there was an irregular nodular mass $(7 \times 8 \mathrm{~cm})$ involving the right ovary and a mild ascites with slightly increased peritoneal enhancement. The skin biopsy showed leucocytoclastic vasculitis with IgM deposits on immunofluorescence study. A coelioscopy disclosed multiple purpuric lesions on the peritoneum, ovaries, and fallopian tubes. The ovarian mass was resected and biopsies of the peritoneum and fallopian tubes were performed. They revealed leucocytoclastic vasculitis with fibrinoid necrosis (figs 1 and 2). Pathological study of the ovarian mass revealed a benign tumour.

The patient was diagnosed as having Waldenström's macroglobulinaemia with mixed cryoglobulinaemia and systemic vasculitis (peritoneal, fallopian tubes, and cutaneous). Cytotoxic treatment was initiated (cyclophosphamide, vincristine, and prednisone) resulting in a good initial response. Abdominal symptoms decreased, and arthralgias and purpura of the legs were absent after two courses of 


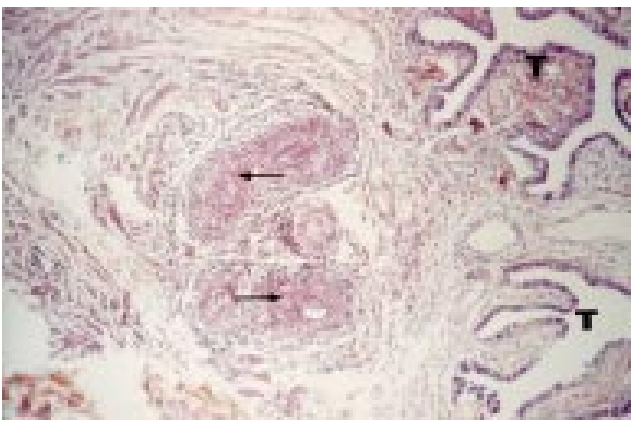

Figure 1 Fallopian tube (T) biopsy specimen showing leucocytoclastic vasculitis with fibrinoid necrosis (arrows). Haematoxylin and eosin stained; magnification, $\times 10$.

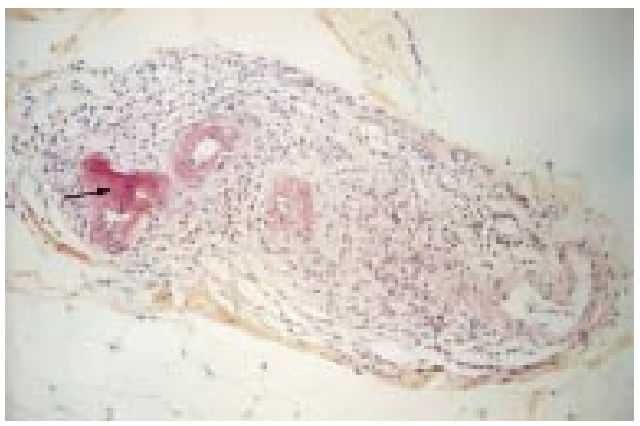

Figure 2 Peritoneal biopsy specimen showing vasculitis of a small blood vessel with fibrinoid necrosis (arrow).

Haematoxylin and eosin stained; magnification, $\times 20$.

chemotherapy. Abdominal CT showed the resolution of the ascites. Ten days after the third course of chemotherapy, a right pneumonia with fever occurred while the patient was leucopenic (white blood cell count, $0.8 \times 10^{9} \%$ litre with $70 \%$ polynuclear neutrophils). Blood cultures were negative but the clinical features suggested a nosocomial infection. Despite the initial administration of broad spectrum antibiotic treatment, haemodynamic instability and respiratory failure ensued and the patient died of multisystem failure. Permission was not obtained for a necropsy.

\section{Discussion}

Waldenström's macroglobulinaemia is an uncommon, low grade, small cell lymphoma with monoclonal IgM production. This protein accounts for the increased plasma viscosity often seen in this disease, and causes some of the most frequent symptoms. Other less common protein complications include cold agglutinin haemolytic anaemia, peripheral neuropathy, amyloidosis, and cryoglobulinaemia. ${ }^{5}$

The term cryoglobulinaemia refers to the presence in the serum of one or more immunoglobulins that precipitate reversibly at a temperature below $37^{\circ} \mathrm{C}$. According to Brouet et al, ${ }^{4}$ cryoglobulins can be classified on the basis of their immunoglobulin composition as type I, consisting of a monoclonal immunoglobulin alone; type II, as a mixture of monoclonal and polyclonal immunoglobulins; and type III, consisting of polyclonal immunoglobulins. They are found in small quantities in normal serum, ${ }^{6}$ but in large concentrations in many pathological conditions. Whereas type III cryoglobulinaemia is mainly noted as the cause of clinical disease in several series, type I and II cryoglobulins are more commonly associated with immunoproliferative disorders. Cryoglobulinaemia in association with Waldenström's macroglobulinaemia occurs in $8-18 \%$ of patients, and consists of either monoclonal IgM (type I) or mixed immunoglobulin complexes in which monoclonal IgM behaves as an antibody against polyclonal IgG (type II), as in our case. Fewer than $5 \%$ of cases of cryoglobulinaemia associated with Waldenström's macroglobulinaemia are symptomatic; they usually involve type I cryoglobulins and cryoglobulinaemia might have an unfavourable role regarding the prognosis of the disease. ${ }^{7}$ Patients with symptomatic cryoglobulinaemia have many different manifestations of vasculitis, including renal involvement with rapidly progressive glomerulonephritis, purpura, leg ulcers, polyarthralgia, Raynaud's phenomenon, liver involvement, and peripheral neuropathy. To our knowledge, vasculitis involving the peritoneum and the fallopian tubes secondary to type II cryoglobulinaemia has not yet been described.

Peritoneal or mesenteric vasculitis has been reported in association with periarteritis nodosa, rheumatoid arthritis, HenochSchoenlein purpura, Wegener's granulomatosis, systemic lupus erythematosus, and ChurgStrauss syndrome, ${ }^{89}$ but never in mixed cryoglobulinaemia. Typical features include diffuse non-specific abdominal pain progressing on occasion to gastrointestinal haemorrhage, perforation, or more rarely infarction. By contrast, isolated cases of ovarian or fallopian tube necrotising vasculitis have been reported both in association with periarteritis nodosa, and as a rare finding in routine gynaecological surgery, especially when this involves the cervix, uterus, or oviduct. ${ }^{10}$ We think that the ascites with slightly increased peritoneal enhancement, and possibly the abdominal pain that occurred in our patient, might be ascribed to the concurrent peritoneal serositis. This notion was strengthened by the simultaneous relief of clinical symptoms and resolution of ascites after treatment.

The pathological appearance of cryoglobulinaemia is a widespread vasculitis involving small and medium sized vessels usually resulting in ischaemia of the skin, nerve, and renal tissue. The cause of this vasculitis remains unclear. Cryoglobulins are thought to form complexes that activate inflammatory effectors and complement factors to induce vascular lesions characterised by neutrophil infiltration, deposition of immune complexes, and necrosis of the vessel wall. ${ }^{11}$

In conclusion, our case suggests that cryoglobulinaemia could be taken into consideration among the aetiologies of peritoneal and fallopian tube vasculitis. Clinicians should be aware of this uncommon complication, particularly in patients with associated lymphoid malignancy.

1 Waldenström J. Incipient myelomatosis or essential hyperglobulinemia with fibrinogenopenia. A new syndrome. Acta Med Scand 1944;117:216-47. 
Wooten MD, Jasin HE. Vasculitis and lymphoproliferative diseases. Semin Arthritis Rheum 1996;26:564-74.

3 Gorevic PD, Kassad HJ, Levo Y, et al. Mixed cryoglobulinemia: clinical aspects and long-term follow up of 40 patients. Am f Med 1980;60:287-308.

4 Brouet JC, Clauvel JP, Danon F, et al. Biologic and clinical significance of cryoglobulins. Am F Med 1974;57:775-88. Dimopoulos MA, Alexanian R. Waldenström's macroglobulinemia. Blood 1994;83:1452-9.

6 Maire MA, Mittey M, Lambert PH. The presence of cryoprecipitable immunoglobulins in normal human sera may reflect specific molecular interactions. Autoimmunity 1989 2:155-64.

7 Gobbi PG, Bettini R, Montecucco C, et al. Study of prognosis in Waldenstrom's macroglobulinemia: a proposal for a simple binary classification with clinical and investigational utility. Blood 1994;83:2939-45.

8 Miescher PA, Huang YP, Izui S. Type II cryoglobulinemia. Semin Hematol 1995;32:80-5.

9 Invernizzi F, Pietrogrande M, Sagramoso B. Classification of the cryoglobulinemic syndrome. Clin Exp Rheumatol 1995;13(suppl):S123-8.

10 Ilbery M, Lyons B, Sundaresan V. Ovarian necrotizing vasculitis causing major intra-abdominal haemorrhage after IVF. Case report and literature review. Br f Obstet Gynaecol 1991;98:596-9.

11 Dammacco F, Sansonno D. Mixed cryoglobulinemia as a model of systemic vasculitis. Clin Rev Allergy Immunol 1997;15:97-119. 